\title{
В.В. Ерохин
}

\section{Управление мощностью в синхронных системах с кодовым разделением каналов на основе оценки времени задержки сигнала}

\begin{abstract}
Разработан оптимальный алгоритм управления мощностью излучения транспондера синхронной системы с кодовым разделением каналов на основе оптимального оценивания времени задержки сигнала. Методами имитационного статистического моделирования показано, что реализация предложенного алгоритма позволяет обеспечить требуемые энергетические характеристики.
\end{abstract}

Ключевые слова: транспондер, управление мощностью, оценка времени задержки сигнала, фильтр Калмана. doi: 10.21293/1818-0442-2016-19-2-31-34

В системах мобильной связи с кодовым разделением каналов (CDMA) применяется управление мощностью сигнала мобильной станции (МС) с целью оптимизации энергетических и эксплуатационных характеристик. Установлено, что различия в уровнях мощности принимаемых сигналов, вызванные неодинаковой удаленностью пользовательских терминалов от базовой станции (БС), приводят к проявлению эффекта «ближний-дальний». В частности, если одна мобильная станция расположена вблизи базовой, а другая находится на границе зоны обслуживания, то разница в уровне принимаемых сигналов может достигать 80 дБ и более. Такое явление свойственно всем технологиям многостанционного доступа, однако в наибольшей степени оно сказывается на работе системы с технологией CDMA, в которой все соседние базовые станции работают на одной частоте. Мощность принимаемого БС сигнала зависит от взаимного расположения базовой и мобильной станций, параметров канала распространения радиоволн и излучаемой мощности [1-5].

Эффект «ближний-дальний» проявляется в том, что приемник захватывает относительно мощный сигнал, и тем самым делает невозможным обнаружение и прием более слабых сигналов. Проблема заключается в ограниченном динамическом диапазоне приемника, что снижает способность по обнаружению слабого сигнала в присутствии более мощного. Данная проблема относится к приемникам, в которых разрешение аналого-цифрового преобразователя (АЦП) ограничивает диапазон обнаруживаемых сигналов. Согласно логике работы в системе автоматической регулировки усиления (АРУ) приемника с приходом мощного сигнала уменьшается коэффициент усиления, чтобы предотвратить насыщение АЦП, который вызывает слабый сигнал, чтобы попасть в динамический диапазон.

В сотовых системах мобильной связи энергетическая проблема «ближний-дальний» решается путем управления мощностью передатчика. При регулировке мощности абонентской станции по замкнутому циклу петля обратной связи определяет значение отношения сигнал/помеха по мощности при помощи пилот-сигнала. Пилот-сигнал проходит через турбулентную среду распространения электромагнитных волн и претерпевает различные искажения $[4,5]$.

В данной работе рассматривается способ управления мощностью на основе оценки времени задержки сигнала в синхронной системе обмена данными (ССОД) с кодовым разделением каналов.

В работах $[2,3]$ показано, что если расстояние между передающей и приемной антеннами увеличивается, то мощность радиосигнала экспоненциально снижается по логарифмическому закону. Мощность принимаемого сигнала в децибелах может быть представлена как функция дальности $d$ :

$$
P_{\text {прм }}(d)=P_{\text {прд }}(u)-20 \lg L-10 \gamma \lg \left(\frac{d}{d_{0}}\right)+n_{p},
$$

где $P_{\text {прд }}(u)$ - управляемая мощность; $d_{0}$ - калибровочное значение дальности; $L-$ калибровочное значение мощности на дальности $d_{0} ; \gamma$ - коэффициент потерь энергии сигнала в среде; $u$ - управляющий сигнал; $n_{p}$ - белый гауссовский шум с нулевым математическим ожиданием и дисперсий $D_{p}$.

В ССОД каждый объект излучает свои сигналы в определенные, известные заранее всем объектам моменты времени. Такая организация работы позволяет любому из объектов, зафиксировав с высокой точностью, определяемой в значительной степени структурой сигнала, момент прихода сообщения, переданного другим объектом, измерить задержку распространения сигнала. Полученное значение задержки отличается от истинного на величину, определяемую рассогласованием временных шкал передающего и принимающего объектов. Применительно к системам, основанным на измерениях с использованием единой шкалы времени, значение дальности, соответствующее измеренной задержке, принято называть псевдодальностью [6].

Для измерения дальностей между объектами применяется беззапросный временной метод. Измеренная псевдодальность $d$ между объектами сети определяется по временной задержке момента $\tau_{i}$ приема сигнала $i$-м объектом относительно априорно известного момента $\tau_{j}$ излучения этого сигнала j-м объектом 


$$
d=c\left(\tau_{i}-\tau_{j}\right)=c \tau,
$$

где $c$ - скорость распространения радиосигнала вдоль радиотрассы.

Таким образом, измеренная дальность между передатчиком и приемником является основной исходной информацией для реализации способа управления мощностью. Значение дальности можно определить путем измерения времени задержки сигнала в каналах обмена данными ССОД.

Рассмотрим задачу оптимального управления мощностью в постановке, когда у объекта, подверженного действию случайных возмущений, нельзя непосредственно измерить состояние, а можно лишь получить оценку переменных состояния с помощью измерительной системы, которая также подвержена действию случайных возмущений, приводящим к ошибкам измерения $[7,8]$. Рассматриваемая система, в которой осуществляются операции управления и оценивания, описывается соотношениями:

$$
\begin{gathered}
\overline{\mathbf{x}}_{v}=\boldsymbol{\Phi}_{v-1} \overline{\mathbf{x}}_{v-1}+\mathbf{B}_{v} \overline{\mathbf{u}}_{v}+\overline{\mathbf{n}}_{x v}, \\
\bar{\xi}_{v}=\mathbf{H}_{v} \overline{\mathbf{x}}_{v}+\overline{\mathbf{n}}_{v},
\end{gathered}
$$

где $v$ - временной индекс; $\boldsymbol{\Phi}_{v}-$ фундаментальная матрица переходов размерности $(n \times n) ; \overline{\mathbf{n}}_{x v}$ и $\overline{\mathbf{n}}_{v}-$ некоррелированные дискретные белые гауссовские шумы (ДБГШ) с нулевыми математическими ожиданиями и ковариационными матрицами $\mathbf{Q}_{v}$ и $\mathbf{V}_{v}$ и размерности $(n \times n)$ и $(m \times m)$ соответственно; $\mathbf{B}_{v}-$ матрица коэффициентов управления; $\overline{\mathbf{u}}_{v}-$ вектор управляющих воздействий.

Так как управление должно удовлетворять условию физической реализуемости, в каждый момент времени $v$, то $\overline{\mathbf{u}}_{v}$ может зависеть только от доступных к данному моменту времени наблюдений, т.е. $\overline{\mathbf{u}}_{v}=f\left(\bar{\xi}_{v-1}\right)$. Функционал качества управления имеет вид $[7,8]$

$$
J=\mathrm{M}\left\{\sum_{v=0}^{N-1}\left[\overline{\mathbf{x}}_{v}^{T} \mathbf{S}_{v} \overline{\mathbf{x}}_{v}+\overline{\mathbf{u}}_{v}^{T} \mathbf{Q}_{v} \overline{\mathbf{u}}_{v}\right]+\left[\overline{\mathbf{x}}_{N}^{T} \mathbf{\Lambda} \overline{\mathbf{x}}_{N}\right]\right\},
$$

где $\mathbf{S}_{v}, \boldsymbol{\Lambda}$ - неотрицательно определенные симметричные матрицы размера $(n \times n)$; $\mathrm{M}\{\}$ - знак математического ожидания. Требуется определить закон управления $\hat{\mathbf{u}}_{v}=\overline{\mathbf{u}}_{v}\left(\bar{\xi}_{1}^{v-1}\right)$, оптимальный по локальному критерию качества $[7,8]$ :

$$
\hat{\overline{\mathbf{u}}}_{v}=\min _{\overline{\mathbf{u}}_{v}}{ }^{-1}\left\{J_{v}\right\},
$$

где

$$
J_{v}=\mathrm{M}\left\{c_{v}\left(\overline{\mathbf{x}}_{v}, \overline{\mathbf{u}}_{v}\right)\right\}=\iint_{\overline{\mathbf{x}} \boldsymbol{\xi}} c_{v}\left(\overline{\mathbf{x}}_{v}, \overline{\mathbf{u}}_{v}\left(\bar{\xi}_{1}^{\nu-1}\right)\right) p\left(\overline{\mathbf{x}}_{v}, \bar{\xi}_{1}^{v-1}\right) d \overline{\mathbf{x}}_{v} d \bar{\xi}_{1}^{v-1}
$$

Здесь $c_{v}\left(\overline{\mathbf{x}}_{v}, \overline{\mathbf{u}}_{v}\right)$ - квадратичная функция потерь.

В (4) усреднение функций стоимости производится по плотностям вероятностей всех случайных величин, а все управления $\hat{\mathbf{u}}_{1}^{\nu-1}$ считаются заданными. Представим совместную плотность вероятно- сти, входящую в минимизируемый функционал (3) в виде произведения

$$
p\left(\overline{\mathbf{x}}_{v}, \bar{\xi}_{1}^{\nu-1}\right)=p\left(\overline{\mathbf{x}}_{v} \mid \bar{\xi}_{1}^{\nu-1}\right) p\left(\bar{\xi}_{1}^{\nu-1}\right) .
$$

Тогда

$$
J_{v}=\int_{\bar{\xi}}\left[\int_{\overline{\mathbf{x}}} c_{v}\left(\overline{\mathbf{x}}_{v}, \overline{\mathbf{u}}_{v}\left(\bar{\xi}_{1}^{v-1}\right)\right) p\left(\overline{\mathbf{x}}_{v} \mid \bar{\xi}_{1}^{\nu-1}\right) d \overline{\mathbf{x}}_{v}\right] p\left(\bar{\xi}_{1}^{\nu-1}\right) d \bar{\xi}_{1}^{\nu-1} .
$$

Так как всегда выполняется условие $p\left(\bar{\xi}_{1}^{\nu-1}\right)>0$, то минимизация показателя качества достигается при минимизации интеграла в квадратных скобках. То есть вместо (4) получаем

$$
\begin{gathered}
\hat{\mathbf{u}}_{v}=\min _{\overline{\mathbf{u}}_{v}}{ }^{-1}\left\{\int_{\overline{\mathbf{x}}} c_{v}\left(\overline{\mathbf{x}}_{v}, \overline{\mathbf{u}}_{v}\left(\bar{\xi}_{1}^{v-1}\right)\right) p\left(\overline{\mathbf{x}}_{v} \mid \bar{\xi}_{1}^{v-1}\right) d \overline{\mathbf{x}}_{v}\right\}= \\
=\min _{\overline{\mathbf{u}}_{v}}{ }^{-1} \mathrm{M}\left\{\left.c_{v}\left(\overline{\mathbf{x}}_{v}, \overline{\mathbf{u}}_{v}\right)\right|_{\left.\bar{\xi}_{1}^{v-1}\right\} .}\right.
\end{gathered}
$$

Для определения плотности вероятности, входящей в (4), используются соотношения оптимальной фильтрации Р.Л. Стратоновича [6]

$$
\left\{\begin{array}{l}
p\left(\overline{\mathbf{x}}_{v} \mid \bar{\xi}_{1}^{v-1}\right)=\int_{\overline{\mathbf{x}}} p\left(\overline{\mathbf{x}}_{v} \mid \overline{\mathbf{x}}_{v-1}, \overline{\mathbf{u}}_{v}\right) p\left(\overline{\mathbf{x}}_{v-1} \mid \bar{\xi}_{1}^{v-1}\right) d \overline{\mathbf{x}}_{v-1}, \\
p_{v}\left(\overline{\mathbf{x}}_{v}\right)=k p\left(\bar{\xi}_{v} \mid \overline{\mathbf{x}}_{v}\right) \tilde{p}_{v}\left(\overline{\mathbf{x}}_{v}\right) .
\end{array}\right.
$$

Совокупность уравнений (5), (6) представляет собой алгоритм решения задачи оптимального управления стохастическими системами по локальному критерию, при этом основной составной задачей оптимального управления является задача оптимальной фильтрации.

Требуется найти управление $\overline{\mathbf{u}}_{v}$ из множества допустимых, обеспечивающее минимум функционала (3). В результате минимизации (3) найдено следующее представление для оптимального управления $[7,8]$ :

$$
\hat{\overline{\mathbf{u}}}_{v}=-\mathbf{L}_{v} \hat{\overline{\mathbf{x}}}_{v-1},
$$

в котором матрица коэффициентов усиления регулятора $\mathbf{L}_{v}$ определяется выражением

$$
\mathbf{L}_{v}=\left(\mathbf{B}_{v}^{T} \mathbf{R}_{v+1} \mathbf{B}_{v}+\mathbf{V}_{v}\right)^{-1} \mathbf{B}_{v}^{T} \mathbf{R}_{v+1} \boldsymbol{\Phi}_{v} .
$$

Матрица $\mathbf{R}_{v}$ находится путем решения матричного уравнения Риккати для дискретного времени

$$
\mathbf{R}_{v}=\boldsymbol{\Phi}_{v}^{T} \mathbf{R}_{v+1} \boldsymbol{\Phi}_{v}+\mathbf{Q}_{v}-\mathbf{L}_{v}^{T}\left[\mathbf{B}_{v}^{T} \mathbf{R}_{v+1} \mathbf{B}_{v}+\mathbf{V}_{v}\right] \mathbf{L}_{v}
$$
при граничном условии $\mathbf{R}_{N}=\boldsymbol{\Lambda}$.

Апостериорная оценка вектора состояния объекта управления по результатам наблюдений удовлетворяет уравнению

$$
\hat{\overline{\mathbf{x}}}_{v}=\boldsymbol{\Phi}_{v} \hat{\overline{\mathbf{x}}}_{v-1}+\mathbf{B}_{v} \overline{\mathbf{u}}_{v}+\mathbf{K}_{v}\left[\bar{\xi}_{v}-\mathbf{H}_{v}\left(\boldsymbol{\Phi}_{v} \hat{\overline{\mathbf{x}}}_{v-1}+\mathbf{B}_{v} \overline{\mathbf{u}}_{v}\right)\right] .
$$

Матрица коэффициентов усиления регулятора $\mathbf{K}_{v}$ рассчитывается по формуле

$$
\mathbf{K}_{v}=\boldsymbol{\Phi}_{v} \tilde{\mathbf{R}}_{v} \mathbf{H}_{v}^{T}\left[\mathbf{H}_{v} \tilde{\mathbf{R}}_{v} \mathbf{H}_{v}^{T}+\mathbf{V}_{v}\right]^{-1},
$$

где $\tilde{\mathbf{R}}_{v}=\boldsymbol{\Phi}_{v} \mathbf{R}_{v-1} \boldsymbol{\Phi}_{v}^{T}+\mathbf{Q}_{v}$ - экстраполированная ковариационная матрица ошибок оценок.

Выражения (9)-(11) являются уравнениями фильтра Калмана для дискретных линейных систем, 
обеспечивающего нахождение оптимальной оценки вектора состояния объекта управления по критерию минимума среднего квадрата ошибки. Соотношения (7), (8) соответствуют случаю определения оптимального управления линейной дискретной детерминированной системой [9].

Структура оптимальной системы, в которой по измерениям производится оценка вектора состояния, используемая в управлении, изображена на рис. 1.

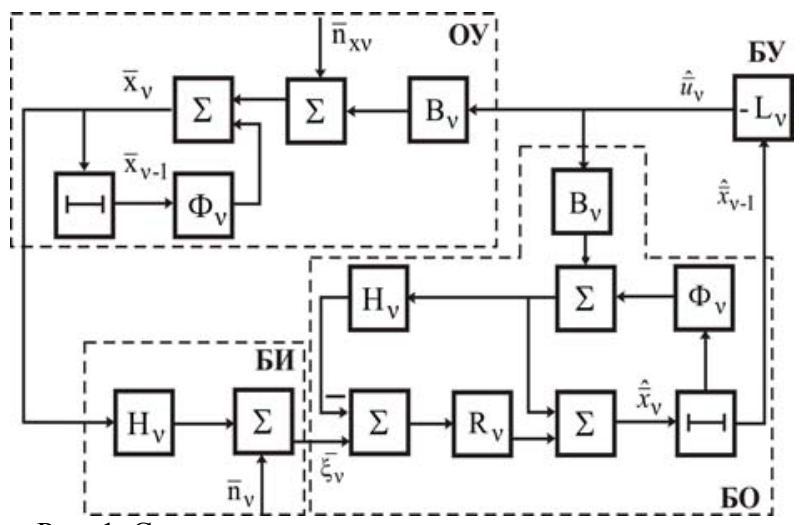

Рис. 1. Структура системы оптимального управления с оценкой состояния: ОУ - объект управления; БУ - блок управления; БИ - блок измерения; БО - блок оценивания

В соответствии с утверждением теоремы разделения или стохастической эквивалентности задачи синтеза оптимального управления и оптимальной фильтрации можно решать независимо (раздельно) $[7,8]$. Для измерения псевдодальности в транспондере ССОД используется оптимальный фильтр второго порядка для следящей системы за задержкой огибающей сигнала [6]. При синтезе сглаживающего фильтра второго порядка априорная модель изменения задержки сигнала задается уравнениями

$$
\begin{aligned}
\tau_{v} & =\tau_{v-1}+T V_{\tau, v-1}, \\
V_{\tau, v} & =V_{\tau, v-1}+n_{\tau, v-1},
\end{aligned}
$$

где $n_{\tau, v}-$ ДБГШ с дисперсией $D_{n_{\tau}}$.

Запишем уравнение наблюдения для следящей системы в виде

$$
\xi_{v}=\tau_{v}+n_{\tau, v},
$$

где $n_{\tau, v}-$ ДБГШ с дисперсией $D_{n}$.

Синтезируем фильтр Калмана, полагая, что модель изменения задержки определяется (12), (13). Уравнения оптимальной фильтрации имеют вид

$$
\begin{gathered}
\hat{\tau}_{v}=\tilde{\tau}_{v-1}+K_{1, v}\left(\xi_{v}-\tilde{\tau}_{v}\right), \\
\tilde{\tau}_{v}=\hat{\tau}_{v-1}+T \hat{V}_{\tau, v-1}, \\
\hat{V}_{v}=\hat{V}_{\tau, v-1}+K_{2, v}\left(\xi_{v}-\tilde{\tau}_{v}\right), \\
K_{1, v}=\frac{R_{11, v}}{D_{n_{\tau}}}, \quad K_{2, v}=\frac{R_{12, v}}{D_{n_{\tau}}},
\end{gathered}
$$

где $R_{i j}$-элементы матрицы $\mathbf{R}$ дисперсий ошибок фильтрации вектора $\overline{\mathbf{x}}=\left|\tau V_{\tau}\right|^{T}$, которая удовлетворяет уравнениям

$$
\begin{gathered}
\mathbf{R}_{x, v}^{-1}=\tilde{\mathbf{R}}_{x, v}^{-1}+\mathbf{H}^{T} \mathbf{H} / D_{n_{\tau}}, \\
\tilde{\mathbf{R}}_{x, v}=\boldsymbol{\Phi} \tilde{\mathbf{R}}_{x, v-1}^{-1} \boldsymbol{\Phi}^{T}+\mathbf{G} \mathbf{G}^{T} D_{w \tau},
\end{gathered}
$$

где $\tilde{\mathbf{R}}_{x, v}-$ матрица дисперсий ошибок экстраполяции вектора $\overline{\mathbf{x}}$;

$$
\boldsymbol{\Phi}=\left|\begin{array}{ll}
1 & T \\
0 & 1
\end{array}\right|, \mathbf{G}=\left|\begin{array}{l}
0 \\
1
\end{array}\right|, \quad \mathbf{H}=\left|\begin{array}{ll}
1 & 0
\end{array}\right| .
$$

В соответствии с выражением (1) и полученным алгоритмом фильтрации вектор состояния в задаче совместного управления и оценивания представим в виде $\overline{\mathbf{x}}=\left|P_{\text {прд }}, \tau, V_{\tau}\right|^{T}$. Реккурентное уравнение динамики управляемой мощности запишем в следующем виде:

$$
P_{\text {прд }, v}=P_{\text {прд }, v-1}+\alpha \cdot \hat{u}_{v},
$$

где $\alpha$ - крутизна регулировочной характеристики.

Предлагаемый алгоритм позволяет выполнять автоматическое регулирование мощности передатчика на основе измерения псевдодальности между абонентами сети.

Одним из возможных путей реализации предлагаемого способа оптимизации энергетических соотношений является управление уровнем сигнала в приемном тракте. Это осуществляется введением закона временной автоматической регулировки усиления (ВАРУ)

$$
u(d) \sim 20 \lg \left(d / d_{\min }\right),
$$

где $d_{\min }-$ начальная дистанция работы ВАРУ (м), рассчитываемая в зависимости от максимальной дистанции и динамического диапазона приёмнопередающего тракта

$$
d_{\min }=d_{\max } / D
$$

где $d_{\max }$ - максимальный диапазон дальности (м); $D$ - динамический диапазон приемного тракта.

Результаты имитационного статистического моделирования алгоритма оценки времени задержки приведены на рис. 2-3, результаты работы алгоритма управления мощностью - на рис. 4 и результаты сравнительного анализа энергетических характеристик без управления и при реализации управления мощностью - на рис. 5. Моделирование проведено при следующих условиях: начальное значение мощности излучаемого сигнала МС -20 дБм, значения параметров были получены путем измерений в системе сотовой связи [3].

Ошибка оценки, $\tau-\hat{\tau}$, нс

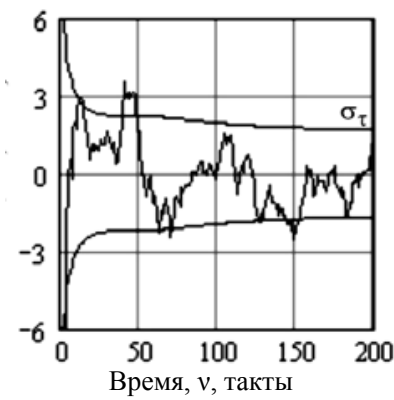

Рис. 2. Ошибка оценки времени задержки сигнала 
Ошибка оценки, $V_{\tau}-\hat{V}_{\tau}, \mu \mathrm{c} / \mathrm{c}$

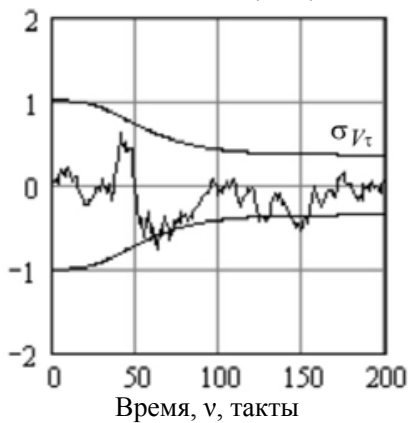

Рис. 3. Ошибка оценки скорости изменения времени задержки сигнала

Излучаемая мощность, $P$, дБм

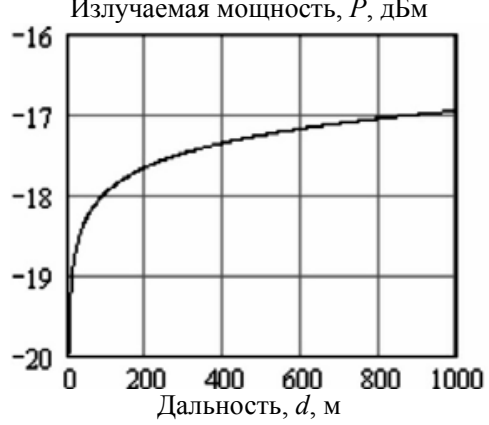

Рис. 4. Зависимость управляемой мощности излучаемого сигнала от дальности

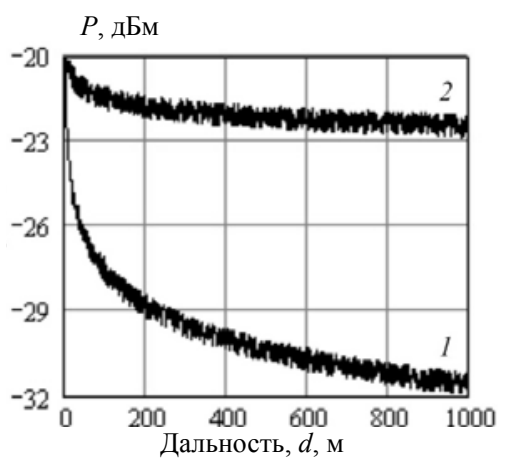

Рис. 5. Зависимость мощности принимаемого сигнала от дальности: 1 - без управления; 2 - с управлением

\section{Выводы}

Синтезированный алгоритм позволяет формировать управляющее воздействие на изменение мощности передатчика в зависимости от дальности между абонентами на основе оценивания с высокой точностью ( $\sigma \approx 2$ нс) времени задержки сигнала. Сравнительный анализ результатов моделирования показал, что реализация предложенного алгоритма позволяет обеспечить требуемые энергетические характеристики между сигналами в ССОД с технологией CDMA.

\section{Литература}

1. Gatsis K. Optimal power management in wireless control systems / K. Gatsis, A. Ribeiro, G.J. Pappas // American Control Conference (ACC) Washington, DC, USA, 2013, June 17-19. - P. 1565-1572.

2. Goldsmith A. Wireless Communications. - New York: Cambridge University Press, 2005. - 561 p.

3. Parsons J.D. The Mobile Radio Propagation Channel. - 2nd ed. - West Sussex: John Wiley \& Sons, 2000.$418 \mathrm{p}$.

4. Коротков Д.А. Алгоритм управления мощностью для системы широкополосного беспроводного доступа стандарта WiMAX IEEE 802.16e / Д.А. Коротков, А.С. Вершинин, Е.П. Ворошилин // Доклады Томского государственного университета систем управления и радиоэлектроники. - 2011. - № 2 (24), ч. 1. - С. 93-97.

5. Шелухин О.И. Управление мощностью мобильной станции в сетях с кодовым разделением каналов на основе оценки ошибки сигнал-помеха / О.И. Шелухин, С.В. Березенко // Электротехнические и информационные комплексы и системы. - 2006. - Т. 2, № 2. - С. 27-29.

6. Тихонов В.И. Статистический анализ и синтез радиотехнических устройств и систем: учеб. пособие для вузов / В.И. Тихонов, В.Н. Харисов. - М.: Радио и связь, 1991. $-668 \mathrm{c}$.

7. Сейдж Э.П. Оптимальное управление системами / Э.П. Сейдж, Ч.С. Уайт. - М.: Радио и связь, 1982. - 392 с.

8. Черноусько Ф.Л. Оптимальное управление при случайных возмущениях / Ф.Л. Черноусько, В.Б. Колмановский. - М.: Наука, 1978. - 352 с.

9. Пантелеев А.В. Теория управления в примерах и задачах / А.В. Пантелеев, А.С. Бортаковский. - М.: Высш. шк., 2003. $-583 \mathrm{c}$.

\section{Ерохин Вячеслав Владимирович}

Канд. техн. наук, доцент каф. авиационного радиоэлектронного оборудования Иркутского филиала Московского гос. технического ун-та гражд. авиации (ИФ МГТУ ГА) Тел.: +7 (395-2) 61-54-10

Эл. почта: Ww_erohin@mail.ru

Erokhin V.V.

Power control in synchronous systems with code channel separation based on evaluation of signal delay

Optimal algorithm based on the estimation of optimal signal delay was developed to control power of transponder radiation of a synchronous system with code channel division. Using the methods of statistical simulation modeling it was shown that the implementation of the proposed algorithm allows to provide the required power characteristics.

Keywords: transponder, power control, evaluation of signal delay time, Kalman filter. 